\title{
Ab Initio Studies on Structural, Elastic, Thermodynamic and Electronic Properties of FeCrAs under Pressures
}

\author{
De-Chun $\mathrm{HE}^{a, *}$, Yong Peng ${ }^{a}$ AND Yong-Wei $\mathrm{HE}^{b}$ \\ ${ }^{a}$ Physical and Mechanical and Electrical Engineering College, Hexi University, Zhangye Gansu, 734000, China \\ ${ }^{b}$ Institute of Atomic and Molecular Physics, Sichuan University, Chengdu 610065, China \\ (Received August 11, 2014; in final form February 10, 2015)

\begin{abstract}
The structural, elastic, thermodynamic and electronic properties of nonmetallic metal FeCrAs are studied within density function perturbation theory. The thermodynamic properties of FeCrAs were deduced based on phonon frequencies within the framework of the quasiharmonic approximation. The calculated elastic modulus under various pressures indicates that FeCrAs is mechanically stable under pressure. The pressure-dependence of bulk and shear modulus, transverse and longitudinal sound velocities $V$ (i.e. $V_{S}$ and $V_{L}$ ), elastic Debye temperature $\Theta_{E}$ of FeCrAs have also been investigated. The calculated values of $B / G$ indicate that FeCrAs presents high ductility under pressure. However, it is interesting that the value of $B / G$ reaches a maximum under $40 \mathrm{GPa}$ and almost remains unchanged when the pressure is above $70 \mathrm{GPa}$. The calculations show that the heat capacity $C_{V}$ of this material is close to the Dulong-Petit limit $3 R$ (about $224.61 \mathrm{~J} \mathrm{~mol}^{-1} \mathrm{~K}^{-1}$ ) at high temperature regime. The analysis of electronic properties find that as the pressure increases, the absolute value of charge for As and Fe atom increases while Cr remains nearly a constant, indicating that the mechanic properties of FeCrAs under pressure should be mostly attributed to the interaction between Fe and As atoms.
\end{abstract}

DOI: $10.12693 /$ APhysPolA.127.1637

PACS: 62.20.de, 65.40.Ba, 65.40.De, 72.15.-v

\section{Introduction}

Iron-pnictide compound FeCrAs, which is famous for its fantastic properties under temperatures and pressures, has attracted much theoretical effort in recent years [1-3]. Under low pressure (below $17 \mathrm{GPa}$ ), FeCrAs exhibits surprising property with Fermi-liquid-like specific heat but the resistivity shows strong non-metallic character and a breakdown of Fermi-liquid behavior. Such phenomenon also exists at quantum critical points of certain second-order phase-transition, such as heavy fermion criticality, Mott criticality and high- $T_{\mathrm{c}}$ cuprate phase transitions [4]. The fantastic properties of FeCrAs can be observed not only under pressures but also at temperatures, demonstrating that the resistivity of Fe$\mathrm{CrAs}$ rises as the temperature falls at low temperature regime, but without a gap opening in the electronic excitation spectrum. Some of these behaviors have been successfully understood in the Kondo systems or disordered metals, which shows weak or strong localization according to whether they have high or low carrier densities. However, for some disordered heavy fermion compounds, such as FeCrAs studied in this work, the behaviors with respect to pressure and temperature are still under investigations.

Considering the exceptional behavior of resistivity for FeCrAs displaying under pressure and temperature, previous effort have been paid the emphasis on the optical, magnetism, and conductive properties of this compound $[2,5,6]$. Wu et al. [1] reported that when the

${ }^{*}$ corresponding author; e-mail: dechunhe@126. com temperature is below $10 \mathrm{~K}$, the resistivity follows a nonFermi-liquid power law while the specific heat shows Fermi-liquid behavior with a large Sommerfeld cofficient. And the in-plane resistivity of $\mathrm{FeCrAs}$ shows an unusual "non-metallic" dependence on temperature $T$, rising continuously with decrease of $T$ from $800 \mathrm{~K}$ to below $100 \mathrm{mK}$. Tafti et al. [2] found that the non-metallic behavior of FeCrAs is shown to be relatively unchanged under the pressure range of 0-17 GPa. Akrap et al. [3] studied the optical properties and electronic structure under ambient conditions and obtained that the optical conductivity has an anisotropic metallic character at room temperature. Specially, they also found that a lowfrequency in-plane phonon mode decreases in frequency for $T<T_{\mathrm{N}}(125 \mathrm{~K})$, suggesting the possibility of spinphonon coupling. Based on the BCS theory and previous investigations, the observing of the soft phonon modes in phonon dispersion curves often has special contribution to the superconductive properties of materials $[7,8]$. Apart from above properties, the elastic, mechanic, lattice dynamic, thermodynamic and electronic properties under pressure and temperature also have crucial application in the further understanding of this material. For example, the study of elastic properties under pressure can provide important help on the further understanding of chemical bonds and cohesion of materials. On the other hand, knowledge of the thermodynamic properties of a crystal can provide essential insight into vibrational properties for the reason that the temperature dependence of heat capacity is governed by details of vibrations of the atoms and could only be determined from experiments for a long time. What is more, the phonon dispersion curves can provide a criterion for crystal dynamical stability and many other physical parameters of mate- 
rials, such as thermodynamic constants, Grüneisen parameter $\gamma$, and superconducting transition temperature $T_{\mathrm{c}}$, can be also obtained from phonon dispersion curves. Thus, the emphases of this work are placed on the elastic, mechanic, thermodynamic and electronic properties of FeCrAs at simultaneously high pressure and temperature.

In this work, our main aim is to investigate elastic, mechanic, thermodynamic and electronic properties of FeCrAs using density functional perturbation theory (DFPT). In Sect. 2 we describe our theoretical methods and the computation details briefly. In Sect. 3, the elastic, mechanic and thermodynamic properties of FeCrAs were deduced based on the obtained phonon frequencies within the framework of the quasiharmonic approximation (QHA). The main results and conclusions are summarized in Sect. 4. All of our work is implemented on Quantum-Espresso (QE) package [9], which is a very useful and credible tool based on the first-principles theory.

\section{Methods and computational details}

The elastic constants of FeCrAs was successfully obtained using the theoretical method proposed by Sin'ko and Smirnov. A brief description of this method is as follows: the lattice vectors $\boldsymbol{a}^{\prime}$ of the strained primitive cell are determined from the lattice vectors $\boldsymbol{a}$ of the equilibrium primitive cell by the relation $\boldsymbol{a}^{\prime}=\boldsymbol{a}\left(I+\widehat{\varepsilon}_{i}\right)$, where $I$ is the unit matrix and $\widehat{\varepsilon}_{i}$ are strain tensors. To calculate five independent constants of hexagonal structures, we considered five independent volume-nonconserving strains, i.e.

$$
\widehat{\varepsilon}_{1}=\left(\begin{array}{ccc}
\gamma & 0 & 0 \\
0 & 0 & 0 \\
0 & 0 & \gamma
\end{array}\right), \widehat{\varepsilon}_{2}=\left(\begin{array}{ccc}
\gamma & 0 & 0 \\
0 & -\gamma & 0 \\
0 & 0 & 0
\end{array}\right)
$$

$\widehat{\varepsilon}_{3}=\left(\begin{array}{lll}\gamma & 0 & 0 \\ 0 & \gamma & 0 \\ 0 & 0 & 0\end{array}\right), \widehat{\varepsilon}_{4}=\left(\begin{array}{lll}0 & 0 & \gamma \\ 0 & 0 & 0 \\ \gamma & 0 & 0\end{array}\right), \widehat{\varepsilon}_{5}=\left(\begin{array}{lll}0 & 0 & 0 \\ 0 & 0 & 0 \\ 0 & 0 & \gamma\end{array}\right)$.

The specific energy of crystal deformed in accord with the $\widehat{\varepsilon}_{1}$ was calculated as a function of the strain magnitude $\gamma$. The values of strain $\gamma$ with the range of $\pm 5 \%$ were used to make the strain energy fit to a second-order polynomial function. Then the strain energy as a function of the strain can be written as

$$
C_{11}+2 C_{13}+C_{33}-2 P=\left.\rho_{1} \frac{\mathrm{d}^{2} E\left(\rho_{1}, \widehat{\varepsilon}_{1}\right)}{\mathrm{d} \gamma^{2}}\right|_{\gamma=0} .
$$

Here $P$ was the pressure applied. Similar results with the order matrix were used in the equation

$$
\begin{aligned}
& 2\left(C_{11}-C_{12}-P\right)=\left.\rho_{1} \frac{\mathrm{d}^{2} E\left(\rho_{1}, \widehat{\varepsilon}_{2}\right)}{\mathrm{d} \gamma^{2}}\right|_{\gamma=0}, \\
& 2\left(C_{11}+C_{12}-P\right)=\left.\rho_{1} \frac{\mathrm{d}^{2} E\left(\rho_{1}, \widehat{\varepsilon}_{3}\right)}{\mathrm{d} \gamma^{2}}\right|_{\gamma=0}, \\
& 4 C_{44}-2 P=\left.\rho_{1} \frac{\mathrm{d}^{2} E\left(\rho_{1}, \widehat{\varepsilon}_{4}\right)}{\mathrm{d} \gamma^{2}}\right|_{\gamma=0},
\end{aligned}
$$

$$
C_{33}-P=\left.\rho_{1} \frac{\mathrm{d}^{2} E\left(\rho_{1}, \widehat{\varepsilon}_{5}\right)}{\mathrm{d} \gamma^{2}}\right|_{\gamma=0} .
$$

The thermodynamic properties of FeCrAs were obtained based on the DFPT. In this method, the Helmholtz free energy of a system can be written as

$$
\begin{aligned}
& F(V, T)=E_{\text {static }}(V)+F_{\mathrm{el}}(V, T)+F_{\mathrm{zp}}(V, T) \\
& \quad+F_{\mathrm{ph}}(V, T),
\end{aligned}
$$

where $E_{\text {static }}(V)$ is the first-principles zero-temperature energy of a static lattice at volume $V$ and $F_{\text {el }}(V, T)$ is the electronic free energy arising from the electronic thermal excitations at certain temperature $T$ and volume $V . \quad F_{\text {el }}(V, T)$ can be evaluated via the standard methods of finite-temperature DFT developed by Mermin (the Fermi-Dirac distribution) [10]:

$$
F_{\text {el }}(V, T)=E_{\text {el }}(V, T)-T S_{e l}(V, T) .
$$

The electronic energy arising from the electronic excitations can be given as

$$
E_{\mathrm{el}}(V, T)=\int n(\varepsilon) f(\varepsilon) \varepsilon \mathrm{d} \varepsilon-\int^{\varepsilon_{\mathrm{F}}} n(\varepsilon) \varepsilon \mathrm{d} \varepsilon,
$$

where $n(\varepsilon)$ is the electronic density of states (EDOS) at the energy eigenvalue $\varepsilon, f$ is the Fermi distribution function, and $\varepsilon_{\mathrm{F}}$ is the energy at the Fermi level.

The electronic entropy is calculated by

$$
S_{e l}(V, T)=-2 k_{\mathrm{B}} \sum_{f_{i}} \ln f_{i}+\left(1-f_{i}\right) \ln \left(1-f_{i}\right) .
$$

Here $k_{\mathrm{B}}$ is the Boltzmann constant.

The term $F_{\mathrm{zp}}(V, T)$ in Eq. (7) is the zero-point motion energy of the lattice given by

$$
F_{\mathrm{zp}}=\frac{1}{2} \sum_{q, j} \hbar \omega_{j}(q, V),
$$

where $\omega_{j}(q, V)$ is the phonon frequency of the $j$-th mode of wave vector $\boldsymbol{q}$ in the first Brillouin zone (BZ).

The last term in Eq. (7) is the phonon free energy due to lattice vibrations, and it can be expressed as

$$
\begin{aligned}
& F_{\mathrm{ph}}(V, T)=k_{\mathrm{B}} T \\
& \quad \times \sum_{q, j} \ln \left(1-\exp \left(-\hbar \omega_{j}(q, V) / k_{\mathrm{B}} T\right) .\right.
\end{aligned}
$$

The calculation of phonon dispersion curves of FeCrAs were calculated within DFPT $[11,12]$ using localdensity approximation [13] with the parametrization of the Perdew and Zunger [14]. A nonlinear core correction to the exchange-correlation energy function was introduced to generate a norm-conserving pseudopotential [15] for $\mathrm{Fe}, \mathrm{Cr}$, and As with the valence electrons configuration $3 \mathrm{~d}^{6} 4 s^{2}, 3 s^{2} 3 p^{6} 3 \mathrm{~d}^{5} 4 s^{1}$, and $4 s^{2} 4 p^{3}$, respectively. To ensure the convergence of the free energy and phonon frequencies, a careful test was made on $k$ and $q$ grids, as well as the kinetic energy cutoff and smearing parameters. For the hexagonal FeCrAs, dynamical matrices were computed at 8 wave $(q)$ vectors using a $4 \times 4 \times 3$ grid in the irreducible wedge of the Brillouin zone. The plane wave cutoff for the wave functions was $60 \mathrm{Ry}$. The Monkhorst-Pack (MP) meshed were $16 \times 16 \times 12$ and the Fermi-Dirac smearing width was $0.02 \mathrm{Ry}$. 


\section{Results and discussion}

\subsection{Structural and elastic properties}

FeCrAs has a hexagonal structure [space group $P \overline{6} m 2$ (No. 187)] and the Fe and Cr atoms occupy the sides $(x, 0,1 / 2)$ and $(z, 0,0)$ with $x=0.2505$ and $z=0.5925$, respectively. The positions of As atoms are at $(0,0,0)$ and $(1 / 3,2 / 3,1 / 2)$. To reach the structure optimization, the volume $V$ and the $c / a$ ratios were optimized. Since the experimental $c / a$ ratio of FeCrAs is about 0.599 [3], a series of different $c / a$ ratio were calculated between 0.50 and 0.70 with a step of 0.001 . For each fixed value of $c / a$, a series of different values of $a$ and $c$ are taken to calculate the total energies $E$ and corresponding volumes $V$. Then an energy-volume $(E-V)$ curve can be obtained by fitting the calculated $E-V$ results to the Birch-Murnaghan equation of states (EOS) [16], in which the pressure-volume relationship expanded to the fourthorder in strain is

$$
\begin{aligned}
P & =3 B_{0} f_{E}\left(1+f_{E}\right)^{5 / 2}\left\{1+\frac{3}{2}\left(B^{\prime}-4\right) f_{E}\right. \\
& \left.+\frac{3}{2}\left[B_{0} B^{\prime \prime}+\left(B^{\prime}-4\right)\left(B^{\prime}-3\right)+\frac{35}{9}\right] f_{E}^{2}\right\},
\end{aligned}
$$

where $f_{E}$ is written as

$$
f_{E}=\left[\left(V_{0} / V\right)^{2 / 3}-1\right] / 2 .
$$

In Fig. 1, we illustrate some of the fitted $E-V$ curves of various $c / a$ ratios. It is found that the $E-V$ curve with the ratio $c / a$ of about 0.597 has the minimum energy, and the corresponding equilibrium lattice parameters $c$ and $a$ are about 3.548 and $5.943 \AA$, respectively. The calculated results combined with experimental data and other theoretical results are given in Table I. Compared with the experimental data [17-19], the calculated lattice parameters are underestimated slightly (about $2.8 \%$ for $c$ and $2.5 \%$ for $a$ ). The difference is reasonable for the reason that experiments are implemented under ambient conditions while the effects of temperature are not

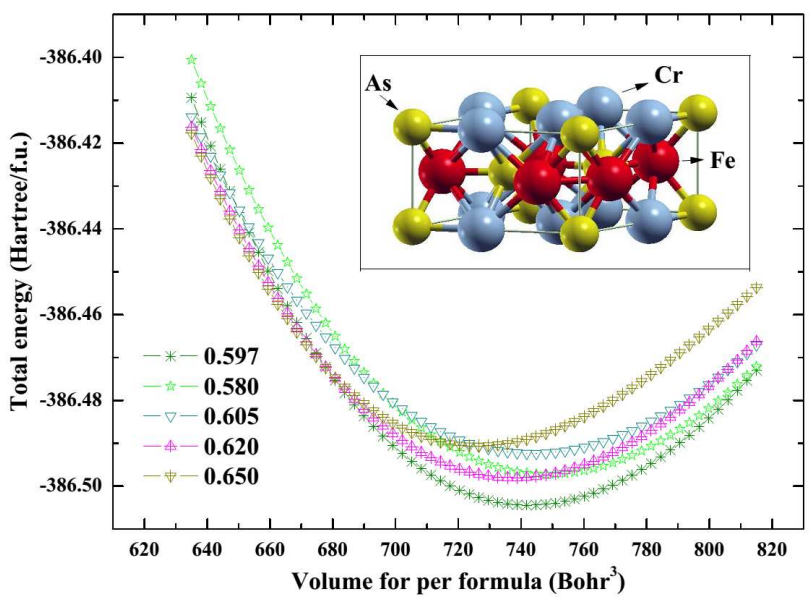

Fig. 1. The total energy $E$ of FeCrAs as a function of primitive cell volume $V$ for different axial ratios $c / a$. The inside graphic is the molecular structure model of FeCrAs.
TABLE I

The calculated structure parameters, bulk modulus $B_{0}$ and its pressure derivation $B_{0}^{\prime}$ at $0 \mathrm{GPa}$ and $0 \mathrm{~K}$ compared with other experimental and theoretical data.

\begin{tabular}{l|c|c|c|c|c}
\hline \hline & $a$ & $c$ & \multirow{2}{*}{$c / a$} & $B_{0}$ & $B_{0}^{\prime}$ \\
\cline { 2 - 3 } \cline { 5 - 6 } & \multicolumn{2}{|c|}{$[\AA]$} & & \multicolumn{2}{|c}{$[\mathrm{GPa}]$} \\
\hline Present work & 5.943 & 3.548 & 0.597 & 228.66 & 4.6547 \\
Theory* $^{*}$ & 5.989 & 3.609 & 0.602 & & \\
Experiment $^{* *}$ & 6.096 & 3.651 & 0.598 & & \\
\hline
\end{tabular}

${ }^{*}$ Ref. [3] GGA using FP-LAPW method by WIEN2k code.

${ }^{* *}$ Ref. $[17-19]$ ambient conditions.

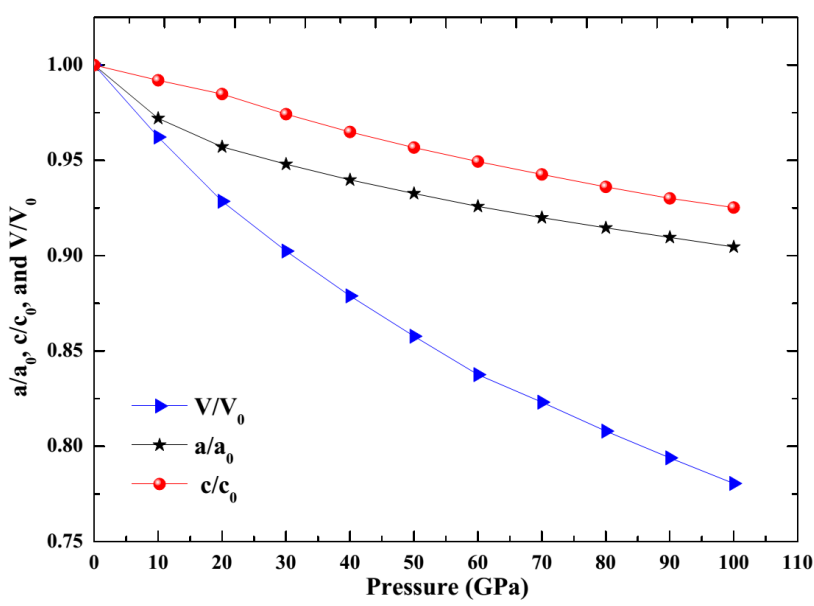

Fig. 2. The normalized parameters $a / a_{0}, c / c_{0}$, and $V / V_{0}$ of FeCrAs as a function of pressure at $0 \mathrm{~K}$.

considered in first-principles calculations. Based on the obtained EOS using Eq. (13), one can obtain the fitted lattice parameters of $c$ and $a$ are about 6.087 and 3.637 at $273 \mathrm{~K}$, agreeing well with experimental results. From Table I, it can be seen that the calculated zero pressure bulk modulus $B_{0}$ and its pressure derivation $B_{0}^{\prime}$ from the Birch-Murnaghan EOS are about 228.66 GPa and 4.6547 at $0 \mathrm{~K}$, respectively. The pressure dependence of the normalized lattice parameters $a / a_{0}, c / c_{0}$, and the normalized primitive cell volume $V / V_{0}$ of FeCrAs (where $a_{0}, c_{0}$, and $V_{0}$ are the zero pressure equilibrium structure parameters) are illustrated in Fig. 2. It is shown that with increasing pressure, the equilibrium ratio $a / a_{0}$ decreases more quickly compared with $c / c_{0}$, indicating that the compression along $a$-axis is larger. In the pressure range of $0-100 \mathrm{GPa}$, the basal plane linear $\mathrm{d}(\ln a) / \mathrm{d} P=0.001003 \mathrm{GPa}^{-1}$ is 1.291 times greater than the interlayer value $\mathrm{d}(\ln c) / \mathrm{d} P=0.000777 \mathrm{GPa}^{-1}$, which suggests that $a$-axis is more sensitive to pressure than the $c$-axis.

\subsection{Elastic properties}

It is known that the elastic constants $C_{11}\left(C_{33}\right)$ are related to the elasticity in length, while the elastic constants $C_{12}\left(C_{13}\right)$ and $C_{44}\left(C_{66}\right)$ represent the elasticity in shape. For FeCrAs, it is found that $C_{11}, C_{12}, C_{13}$, and $C_{33}$ are susceptible to pressure, while the variation of $C_{44}$ 
and $C_{66}$ are slow with increasing pressure (Fig. 3). However, there exists a minimum under $40 \mathrm{GPa}$ for $C_{66}$. The calculations also indicate that the atomic bonds along (001) plane between nearest neighbors are stronger than those along (100) plane. Another striking feature of the elastic constants for FeCrAs is that $C_{11}, C_{12}, C_{13}$, and $C_{33}$ present fluctuate characters under pressure. Based on the obtained elastic constants, the bulk modulus along $a$-axis $\left(B_{a}\right)$ and $c$-axis $\left(B_{c}\right)$ can be defined as follows to qualify the mechanical anisotropy of FeCrAs [20] i.e.:

$$
\begin{aligned}
& B_{a}=a \frac{\mathrm{d} P}{\mathrm{~d} a}=\frac{\Lambda}{2+\alpha}, \\
& B_{c}=c \frac{\mathrm{d} P}{\mathrm{~d} c}=\frac{B_{a}}{\alpha}, \\
& \Lambda=2\left(C_{11}+C_{12}\right)+4 C_{13} \alpha+C_{33} \alpha^{2}, \\
& \alpha=\frac{C_{11}+C_{12}-2 C_{13}}{C_{33}-C_{13}} .
\end{aligned}
$$

The five independent elastic constants combined with bulk modulus $B_{a}$ and $B_{c}$ under pressures are given in Table II. It should be noted that $B_{c}$ is much larger than $B_{a}$ under $0 \mathrm{GPa}$ and above ambient pressure, $B_{a}$ and $B_{c}$ present an increasing trend with pressure. However, $B_{a}$ becomes higher than $B_{c}$ in the pressure range of $10-$ $60 \mathrm{GPa}$. Then $B_{a}$ reaches a maximum with $2191.8 \mathrm{GPa}$ under $90 \mathrm{GPa}$, while the maximum of $B_{c}$ is $2546.1 \mathrm{GPa}$ when pressure is $70 \mathrm{GPa}$. For a hexagonal structure, the mechanical stability under isotropic pressures can be estimated from criterion as follows [21]:

$$
\widetilde{C}_{44}>0, \widetilde{C}_{11}>\left|\widetilde{C}_{12}\right|, \widetilde{C}_{33}\left(\widetilde{C}_{11}+\widetilde{C}_{12}\right)>2 \widetilde{C}_{13}
$$

in which $\widetilde{C}_{\alpha \alpha}=C_{\alpha \alpha}-P(\alpha=1,4), \widetilde{C}_{12}=C_{12}+P$, $\widetilde{C}_{13}=C_{13}+P$. The elastic constants of FeCrAs given in Table II all satisfy these stability conditions, indicating the mechanical stable of FeCrAs.

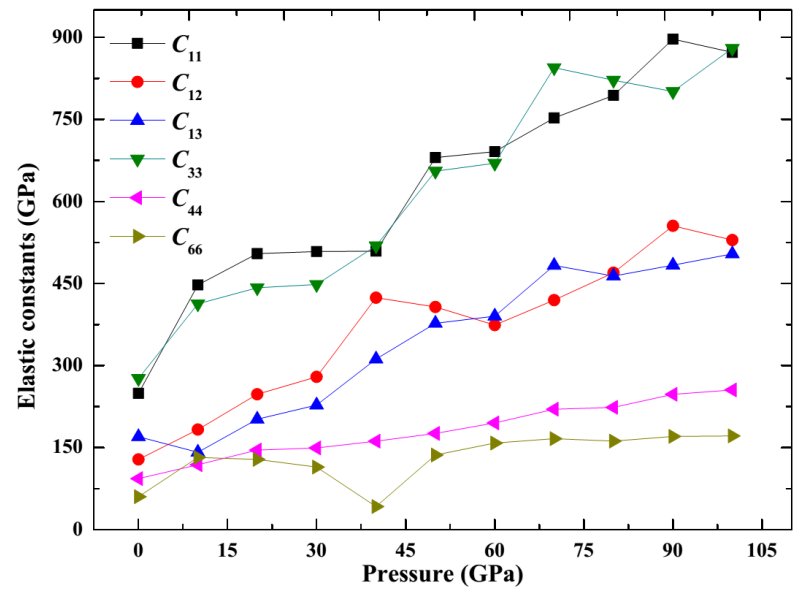

Fig. 3. Pressure dependence of elastic constants of FeCrAs at $0 \mathrm{~K}$.

The isotropic bulk modulus $B_{V}$ and shear modulus $G_{V}$ for a hexagonal crystal can be expressed as
TABLE II

Calculated elastic constants $C_{i j}$ [GPa] of FeCrAs in the pressure range of $0-100 \mathrm{GPa}$, as well as bulk modulus $B_{a}$ and $B_{c}$

\begin{tabular}{c|c|c|c|c|c|c|c|c}
\hline \hline$P$ & $C_{11}$ & $C_{12}$ & $C_{13}$ & $C_{33}$ & $C_{44}$ & $C_{66}$ & $B_{a}$ & $B_{c}$ \\
\hline 0 & 249.3 & 128.5 & 170.1 & 276.5 & 93.5 & 60.4 & 437.9 & 1239.2 \\
10 & 447.6 & 183.1 & 141.7 & 413.1 & 118.9 & 132.2 & 812.0 & 634.6 \\
20 & 504.8 & 247.7 & 202.1 & 442.4 & 145.6 & 128.5 & 1045.4 & 721.3 \\
30 & 508.6 & 279.4 & 227.9 & 448.3 & 149.5 & 114.6 & 1131.5 & 750.7 \\
40 & 509.2 & 424.2 & 312.0 & 519.0 & 161.9 & 42.5 & 1399.7 & 936.5 \\
50 & 680.4 & 407.2 & 377.4 & 655.6 & 176.1 & 136.6 & 1539.1 & 1286.6 \\
60 & 691.3 & 374.2 & 390.4 & 670.2 & 195.4 & 158.6 & 1462.7 & 1437.6 \\
70 & 752.1 & 419.7 & 483.2 & 844.8 & 220.4 & 166.5 & 1446.3 & 2546.1 \\
80 & 794.1 & 469.9 & 463.8 & 821.8 & 223.8 & 162.1 & 1699.8 & 1808.9 \\
90 & 896.9 & 555.6 & 483.7 & 801.1 & 247.7 & 170.6 & 2191.8 & 1434.1 \\
100 & 872.7 & 529.6 & 504.3 & 880.1 & 255.6 & 171.6 & 1930.6 & 1842.8
\end{tabular}

TABLE III

Calculated bulk modulus B [GPa], shear modulus G [GPa], sound velocities $V_{S}, V_{L}, V_{M}[\mathrm{~m} / \mathrm{s}]$ and elastic Debye temperature $\Theta_{E}$ of FeCrAs under pressure.

\begin{tabular}{c|c|c|c|c|c|c|c}
\hline \hline$P$ & $B$ & $G$ & $B / G$ & $V_{S}$ & $V_{L}$ & $V_{M}$ & $\Theta_{E}$ \\
\hline 0 & 186.1 & 66.59 & 2.7947 & 2423.8 & 4924.2 & 2721.5 & 349.4 \\
10 & 247.6 & 129.6 & 1.9105 & 3266.3 & 5882.4 & 3637.9 & 478.0 \\
20 & 303.1 & 136.9 & 2.2140 & 3301.4 & 6217.3 & 3689.3 & 490.2 \\
30 & 322.6 & 130.3 & 2.4758 & 3173.1 & 6192.4 & 3554.3 & 477.0 \\
40 & 400.5 & 89.8 & 4.4599 & 2598.8 & 6254.5 & 2940.1 & 398.2 \\
50 & 481.5 & 153.7 & 3.1327 & 3359.0 & 7099.1 & 3779.5 & 515.9 \\
60 & 484.7 & 168.3 & 2.8799 & 3476.4 & 7135.9 & 3905.7 & 537.1 \\
70 & 563.2 & 183.3 & 3.0726 & 3591.4 & 7539.0 & 4039.6 & 559.2 \\
80 & 578.2 & 187.4 & 3.0854 & 3597.9 & 7563.3 & 4047.2 & 563.8 \\
90 & 621.8 & 201.5 & 3.0859 & 3699.1 & 7772.9 & 4160.9 & 582.9 \\
100 & 633.5 & 205.6 & 3.0812 & 3706.1 & 7786.3 & 4168.9 & 587.3
\end{tabular}

TABLE IV

Mulliken populations of FeCrAs under different pressures.

\begin{tabular}{c|c|c|c|c|c|c}
\hline $\begin{array}{c}\text { Pressure } \\
\text { [GPa] }\end{array}$ & Atoms & $s$ & $p$ & $d$ & Total & $\begin{array}{c}\text { Charge } \\
\text { transfer [e] }\end{array}$ \\
\hline \multirow{3}{*}{0} & $\mathrm{Cr}$ & 2.59 & 6.90 & 5.15 & 14.65 & -0.65 \\
& $\mathrm{Fe}$ & 0.48 & 0.72 & 6.97 & 8.17 & -0.17 \\
& $\mathrm{As}$ & 0.83 & 3.35 & 0.00 & 4.18 & 0.82 \\
\hline \multirow{3}{*}{30} & $\mathrm{Cr}$ & 2.61 & 6.88 & 5.18 & 14.68 & -0.68 \\
& $\mathrm{Fe}$ & 0.49 & 0.71 & 6.99 & 8.18 & -0.18 \\
& $\mathrm{As}$ & 0.78 & 3.35 & 4.13 & 4.13 & 0.87 \\
\hline \multirow{3}{*}{60} & $\mathrm{Cr}$ & 2.63 & 6.83 & 5.22 & 14.68 & -0.68 \\
$\mathrm{As}$ & $\mathrm{Fe}$ & 0.51 & 0.68 & 7.02 & 8.21 & -0.21 \\
\hline \multirow{3}{*}{90} & 0.73 & 3.35 & 0.00 & 4.08 & 0.92 & \\
\hline & $\mathrm{Cr}$ & 2.65 & 6.76 & 5.25 & 14.67 & -0.67 \\
& $\mathrm{Fe}$ & 0.54 & 0.66 & 7.04 & 8.24 & -0.24 \\
& & 0.70 & 3.35 & 0.00 & 4.05 & 0.95
\end{tabular}




$$
\begin{aligned}
& B_{V}=\frac{1}{9}\left[2\left(C_{11}+C_{12}\right)+C_{33}+4 C_{13}\right], \\
& G_{V}=\frac{1}{30}\left(C_{11}+C_{12}+2 C_{33}-4 C_{13}+12 C_{44}+12 C_{66}\right) .
\end{aligned}
$$

The isotropic bulk modulus $B_{R}$ and shear modulus $G_{R}$ are given by:

$$
\begin{aligned}
& B_{R}=\frac{\left(C_{11}+C_{12}\right) C_{33}-2 C_{13}^{2}}{C_{11}+C_{12}+2 C_{33}-4 C_{13}}, \\
& G_{R}=\frac{5}{2}\left\{\left[\left(C_{11}+C_{12}\right) C_{33}-2 C_{13}^{2}\right]^{2} C_{44} C_{66}\right\} / \\
&\left\{3 B_{V} C_{44} C_{66}+\left[\left(C_{11}+C_{12}\right) C_{33}-2 C_{13}^{2}\right]^{2}\left(C_{44}+C_{66}\right)\right\} .
\end{aligned}
$$

Here

$$
C_{66}=\frac{1}{2}\left(C_{11}-C_{12}\right)
$$

The average of the Voigt and Reuss bulk modulus is called as the Voigt-Reuss-Hill (VRH) average and can be commonly used to estimate elastic modulus of polycrystals. The VRH average for shear modulus $(G)$ and bulk modulus $(B)$ are defined as:

$$
G=\frac{1}{2}\left(G_{R}+G_{V}\right), \quad B=\frac{1}{2}\left(B_{R}+B_{V}\right) .
$$

Then the values of the average transverse and longitudinal sound velocities can be calculated from the Navier equation [22]:

$$
V_{S}=\sqrt{\frac{G}{\rho}}, \quad V_{L}=\sqrt{\frac{B+\frac{4}{3} G}{\rho}} .
$$

Based on above sound velocities, the elastic Debye temperature $\Theta_{E}$ can be obtained from the average sound velocity $V_{m}[23]$ :

$$
\Theta_{E}=\frac{h}{k}\left[\frac{3 n}{4 \pi}\left(\frac{\rho N_{a}}{M}\right)\right]^{\frac{1}{3}} V_{m} .
$$

Where $h$ is the Planck constant, $k$ is the Boltzmann constant, $N_{a}$ is the Avogadro number, and $n$ is the number of atoms per formula unit, $M$ is the molecular mass per formula unit. $\rho$ is the density and $V_{M}$ can be obtained by [23]:

$$
V_{M}=\left[\frac{1}{3}\left(\frac{2}{V_{S}^{3}}+\frac{1}{V_{L}}\right)\right]^{-\frac{1}{3}} .
$$

The calculated results for bulk modulus $B$ [GPa], shear modulus $G[\mathrm{GPa}]$, and sound velocities $V_{S}, V_{L}, V_{M}$, as well as elastic Debye temperature $\Theta_{E}$ of FeCrAs under pressure are illustrated in Table III. Generally, the bulk modulus and shear modulus are used to measure the hardness of materials. In the studied pressure range, one can find that the bulk modulus $B$ increases gradually with the increase of pressure, indicating that FeCrAs becomes more and more difficult to compress with increasing pressure. It should be noticed here FeCrAs has a relative high bulk modulus (above $400 \mathrm{GPa}$ when $P>40 \mathrm{GPa}$ ) under pressure since it is known that the bulk modulus of diamond is $434 \mathrm{GPa}$ under ambient conditions [24]. Simultaneously, the ratio of bulk modulus and shear modulus, i.e. $B / G$, has been applied to estimate the ductile or brittle properties of materials. The value of $B / G$ that separates ductile and brittle of mate- rials is about 1.75 . In Table III, the calculated values of $B / G$ are all higher than above value, indicating that FeCrAs presents high ductility under pressure. However, it is interesting that the value of $B / G$ reaches a maximum under $40 \mathrm{GPa}$ and almost remains unchanged when the pressure is above of $70 \mathrm{GPa}$. This phenomenon demonstrates that the pressure can improve a material's ductility in a certain pressure range.

\subsection{Thermodynamic properties}

The accurate thermodynamic properties as a function of pressure and temperature can directly provide the valuable information for understanding the dynamical response of materials under extreme conditions. The thermodynamic properties of $\mathrm{FeCrAs}$ were obtained within the quasi-harmonic approximation (QHA) theory. In the framework of QHA theory, the Helmholtz free energy as a function of volume $V$ and temperature $T$ can be derived after taking into account the thermal electronic excitation and phonon contributions. In order to deduce the thermodynamic properties, we fitted a fourth-order finite-strain EOS [25] to the calculated free energy versus volume at each temperature. The fitted isothermal compressional curves are given in Fig. 4 . It can be seen that, as the pressure increases, the relative volume $V / V_{0}$ decreases at a given temperature, and the relative volume $V / V_{0}$ of higher temperature is less than that of lower temperature at the same pressure. On the other hand, the volume $V$ decreases with the increasing pressure $P$, and increases with the elevation of temperature $T$, indicating that the effect of increasing pressure on FeCrAs is the same as decreasing temperature.

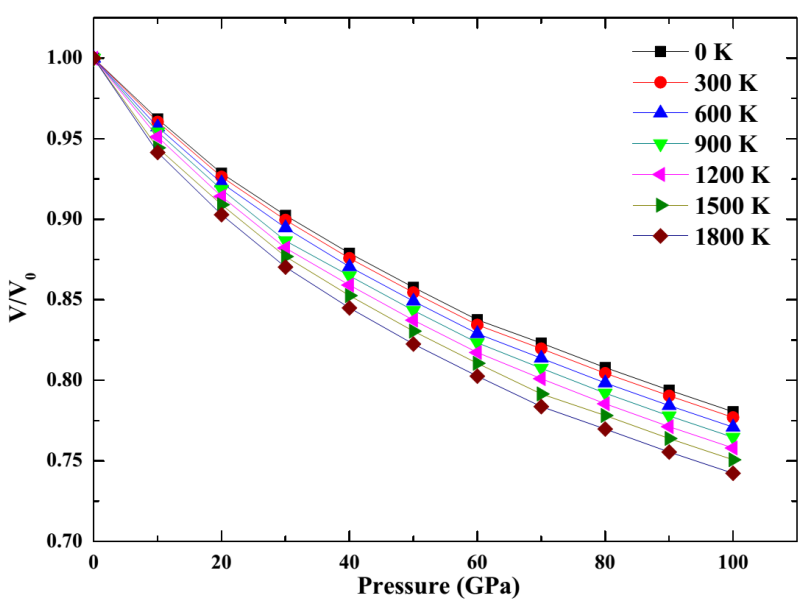

Fig. 4. The isothermal compressional curves at different temperatures. $V_{0}$ denotes the incipient volume of $\mathrm{FeCrAs}$ at the pressure of $0 \mathrm{GPa}$.

The thermal expansion coefficient

$$
\alpha_{V}=\frac{1}{V}\left(\frac{\partial V}{\partial T}\right)_{P}
$$

can be obtained from the equilibrium volume variation with respect to the temperature at each pressure. In Fig. 5a and b, the thermal expansion coefficient as functions of temperature and pressure are plotted. At a given 

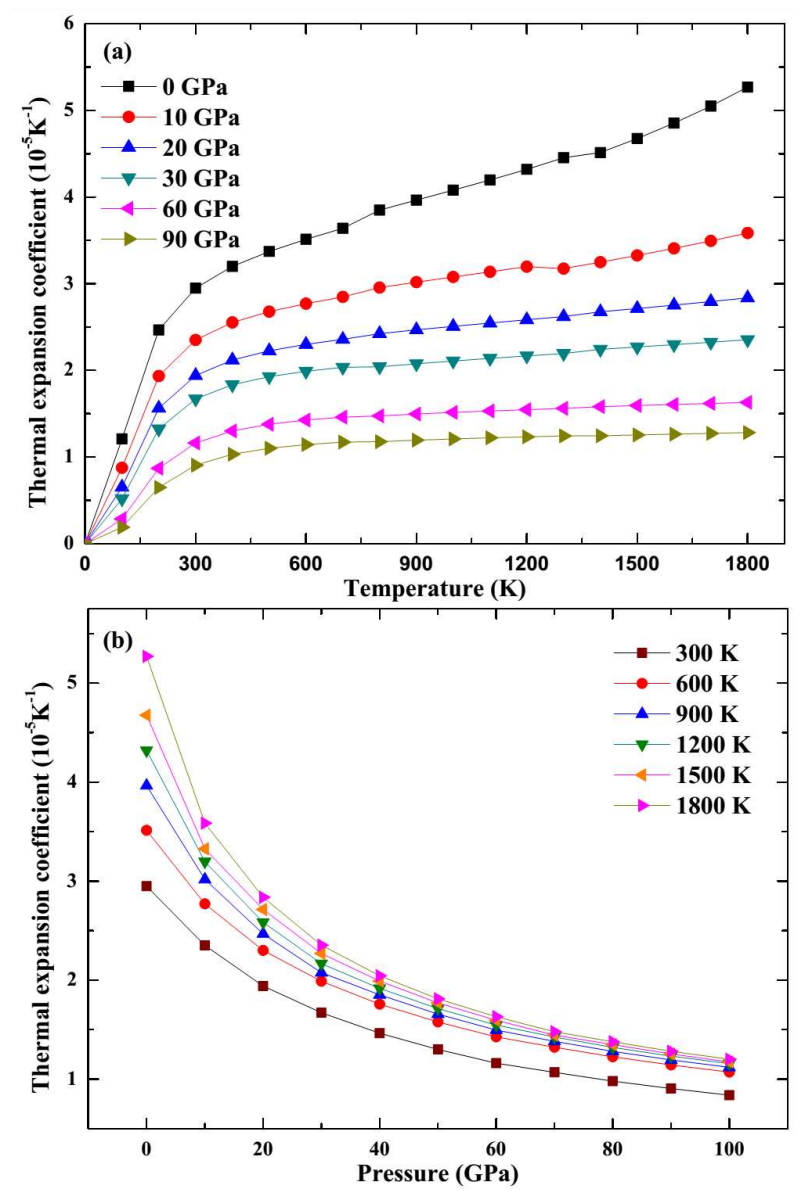

Fig. 5. Thermal expansion coefficient versus temperature (a) and pressure (b) of FeCrAs.

pressure, $\alpha$ increases exponentially at low temperatures and gradually approaches a linear increase at high temperature range. As the pressure increases, the increase of $\alpha$ with temperature becomes smaller, especially at high temperatures. However, $\alpha$ decreases drastically with the increase of pressure at a given temperature. On the other hand, the difference of thermal expansion $\alpha$ at high temperature tends to be smaller compared with that of at low temperature and the curves seem to be parallel to each others, which means that the temperature-dependence of thermal expansion coefficient is small at high temperature.

The specific heat at constant volume is defined as

$$
C_{V}=\left(\frac{\partial U}{\partial T}\right)_{V}
$$

where $U$ is the internal energy of the whole system. The specific heat at constant pressure $C_{P}$ is different from $C_{V}$ due to the thermal expansion caused by anharmonic effects. The relation between $C_{P}$ and $C_{V}$ can be described by the following relations:

$$
C_{P}-C_{V}=\alpha_{V}(T) B_{0} V T \text {. }
$$

Here $\alpha_{V}$ is the volume thermal expansion coefficient. $B_{0}$ is the bulk modulus and $T$ is the temperature, while $V$ is the volume. It is known that $C_{V}$ increases dramati-

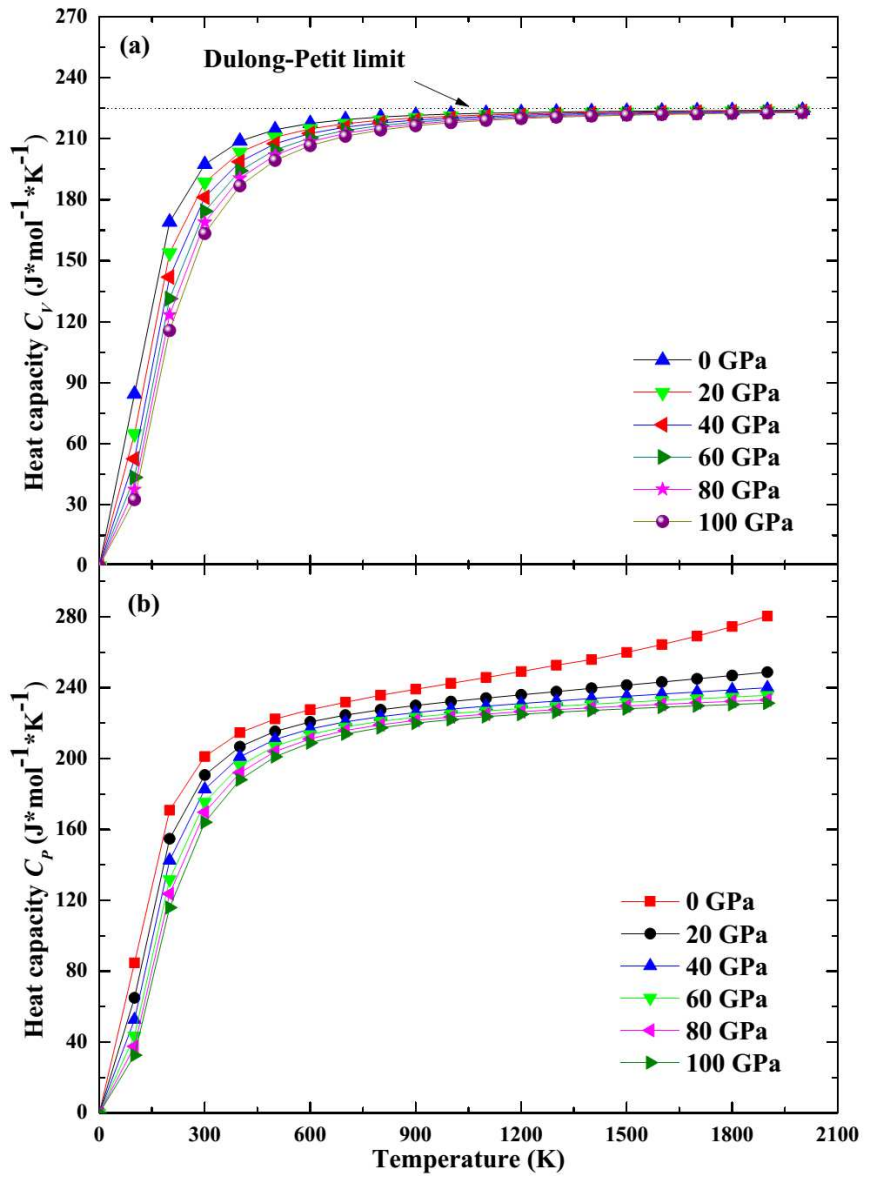

Fig. 6. Calculated constant volume heat capacity $C_{v}$ (a) and $C_{p}$ (b) of FeCrAs as a function of temperature under different pressures. The dotted line displayed in (a) represents Dulong-Petit limit.

cally as temperature increases and finally approaches a constant $3 R$. From Eq. (31), there can be seen the $C_{P}$ of $\mathrm{FeCrAs}$ as a function of temperature at different pressures. As far as we know, there are few studies have been reported on the $C_{V}$ and $C_{P}$ of FeCrAs, thus we present our calculations in Fig. 6 . When $T<1200 \mathrm{~K}$, the heat capacity $C_{V}$ is dependent on both temperature $T$ and pressure $P$. These relations can be attributed to the effect of anharmonic approximations for materials. However, the heat capacity $C_{V}$ is close to the Dulong-Petit limit $3 R$ (about $224.61 \mathrm{~J} \mathrm{~mol}^{-1} \mathrm{~K}^{-1}$ ) at higher temperatures $(>1200 \mathrm{~K})$, presenting a common phenomenon to all solids at high temperatures. On the other hand, the heat capacity decreases more quickly with increasing pressure at low temperature compared with that at high temperature. At a given pressure, $C_{P}$ increases exponentially at low temperatures and gradually turns to a line-increase mode at high-temperature regime. As the pressure increases, $C_{P}$ decreases at a given temperature.

\subsection{Electronic properties under pressure}

To investigate the pressure dependence of electronic properties for FeCrAs, the Mulliken charges [26] are calculated under different pressures. The Mulliken charge 
$Q_{m}(A)$ of $A$ atom and the overlap population $n_{m}(A B)$ for $A-B$ bond are defined as follows:

$$
\begin{aligned}
& Q_{m}(A)=\sum_{k} W(k) \sum_{v}^{A} P_{u v}(k) S_{u v}(k), \\
& n_{m}(A B)=\sum_{k} W(k) \sum_{u}^{A} \sum_{v}^{B} P_{u v}(k) S_{u v}(k) .
\end{aligned}
$$

Here $P_{u v}(k)$ and $S_{u v}(k)$ are the density matrix and the overlap matrix, respectively. $u$ and $v$ are the orbitals on $A$ and $B$, respectively. $W(k)$ is the weight associated the calculated $k$ points in the Brillouin zone. Generally, the magnitude and sign of $Q_{m}(A)$ characterized the iconicity of $A$ atom in the crystal, and $n_{m}(A B)$ can be used to measure the covalent bonding strength approximately. The calculated Mulliken populations of FeCrAs obtained under different pressures are listed in Table IV. From Table IV, it can be deduced that As loses electrons while $\mathrm{Fe}$ and $\mathrm{Cr}$ present negative values under pressures. As the pressure increases, the absolute value of charge for $\mathrm{As}$ and $\mathrm{Fe}$ atom increases while $\mathrm{Cr}$ remains nearly a constant. This indicates that the mechanic properties of FeCrAs under pressure should be mostly attributed to the interaction between $\mathrm{Fe}$ and As atoms.

\section{Conclusions}

In summary, the structural, elastic, thermodynamic and electronic properties of the novel nonmetallic metal FeCrAs under high pressure are investigated within density function theory. The pressure-dependence of the elastic constants, elastic modulus, transverse and longitudinal sound velocities $V$ (i.e. $V_{S}$ and $V_{L}$ ), and elastic Debye temperature $\Theta_{E}$ of FeCrAs have also been obtained firstly. The calculated elastic modulus at various pressures indicates FeCrAs is mechanically stable under pressure. It is also reasonable to deduce that FeCrAs has a relative high bulk modulus under pressure based on the knowledge that the bulk modulus of diamond is $434 \mathrm{GPa}$ under ambient conditions. The calculated values of $B / G$ indicate that FeCrAs presents high ductility under pressure. However, it is interesting that the value of $B / G$ reaches a maximum under $40 \mathrm{GPa}$ and almost remains unchanged when the pressure is above of $70 \mathrm{GPa}$. The calculations show that the heat capacity $C_{V}$ of this material is close to the Dulong-Petit limit $3 R$ (about $224.61 \mathrm{~J} \mathrm{~mol}^{-1} \mathrm{~K}^{-1}$ ) at high temperature regime. The analysis of electronic properties finds that as the pressure increases, the absolute value of charge for As and Fe atom increases while $\mathrm{Cr}$ remains nearly a constant, indicating that the mechanic properties of FeCrAs under pressure should be mostly attributed to the interaction between $\mathrm{Fe}$ and As atoms.

\section{Acknowledgments}

We acknowledge the support for the computational resources by the State Key Laboratory of Polymer Materials Engineering of China in Sichuan University.

\section{References}

[1] W. Wu, A. McCollam, I. Swainson, P.M.C. Rourke, D.G. Rancourt, S.R. Julian, Euro. Phys. Lett. 85, 17009 (2009).

[2] F.F. Tafti, W. Wu, S.R. Julian, J. Phys. Condens. Matter 25, 385601 (2013).

[3] A. Akrap, Y.M. Dai, W. Wu, S.R. Julian, C.C. Homes, Phys. Rev. B 89, 125115 (2014).

[4] T. Senthil, Phys. Rev. B 78, 035103 (2008).

[5] J.G. Rau, H.Y. Kee, Phys. Rev. B 84, 104448 (2011).

[6] J.M. Florez, P. Vargas, C. Garcia, C.A. Ross, J. Phys. Condens. Matter 25, 226004 (2013).

[7] J.S. Tse, Y. Yao, K. Tanaka, Phys. Rev. Lett. 98, 117004 (2007).

[8] Y.K. Wei, N.N. Ge, G.F. Ji, X.R. Chen, L.C. Cai, S.Q. Zhou, D.Q. Wei, J. Appl. Phys. 114, 114905 (2013).

[9] P. Giannozzi, S. Baroni, N. Bonini, M. Calandra, R. Car, C. Cavazzoni, D. Ceresoli, G.L. Chiarotti, M. Cococcioni, I. Dabo, A. Dal Corso, S. de Gironcoli, S. Fabris, G. Fratesi, R. Gebauer, U. Gerstmann, C. Gougoussis, A. Kokalj, M. Lazzeri, L. Martin-Samos, N. Marzari, F. Mauri, R. Mazzarello, S. Paolini, A. Pasquarello, L. Paulatto, C. Sbraccia, S. Scandolo, G. Sclauzero, A.P. Seitsonen, A. Smogunov, P. Umari, R.M. Wentzcovitch, $J$. Phys. Condens. Matter 21, 395502 (2009).

[10] N.D. Mermin, Phys. Rev. 137, A1441 (1965).

[11] S. Baroni, P. Giannozzi, A. Testa, Phys. Rev. Lett. 58, 1861 (1987).

[12] S. Baroni, S.D. Gironcoli, A.D. Corso, P. Giannozzi, Rev. Mod. Phys. 73, 515 (2001).

[13] W. Kohn, L.J. Sham, Phys. Rev. B 140, A1133 (1965).

[14] J.P. Perdew, A. Zunger, Phys. Rev. B 23, 5048 (1981).

[15] B. Hammer, I.B. Hansen, J.K. Norskov, Phys. Rev. B 59, 7413 (1999).

[16] F. Birch, Phys. Rev. 71, 809 (1947).

[17] L. Hollan, Ann. Chim. (Paris) 1, 437 (1966).

[18] M. Nylund, M. Roger, J. Sénateur, R. Fruchart, J. Solid State Chem. 4, 115 (1972).

[19] R. Guérin, M. Sergent, Mater. Res. Bull. 12, 381 (1977).

[20] P. Ravindran, L. Fast, P.A. Korzhavyi, B. Johnnsson, J. Wills, O. Eriksson, J. Appl. Phys. 84, 4891 (1998).

[21] G.V. Sin'ko, N.A. Smirnov, J. Phys. Condens. Matter 14, 6989 (2002).

[22] R. Hill, Proc. Soc. Lond. A 65, 349 (1952).

[23] O.L. Anderson, J. Phys. Chem. Solids 21, 909 (1963).

[24] K.B. Panda, K.S. Ravi Chandran, Comput. Mater. Sci. 35, 134 (2006).

[25] F. Birch, J. Geophys. Res. 91, 4949 (1986).

[26] R.S. Mulliken, J. Chem. Phys. 45, 1833 (1955). 